\title{
Public Engagement in a Global Pandemic
}

\author{
Greg Corbett ${ }^{1, *}$, Ian Collier ${ }^{1}$, Sophy Palmer ${ }^{1}$, Tom Dack ${ }^{1}$ and Xin Ran Liu ${ }^{2}$ \\ ${ }^{1}$ Science and Technology Facilities Council, Scientific Computing, Didcot, United Kingdom \\ ${ }^{2}$ The University of Edinburgh, School of Physics and Astronomy, Edinburgh, United Kingdom
}

\begin{abstract}
UKRI/STFC's Scientific Computing Department (SCD) has a long and rich history of delivering face to face public engagement and outreach, both on site and in public places, as part of the wider STFC programme. Due to the global COVID-19 pandemic, SCD was forced to abandon an extensive planned programme of public engagement, alongside altering the day-to-day working methods of the majority of its staff. SCD had to respond rapidly to create a new, remote only, programme for the summer and for the foreseeable future. This was initially an exercise in improvisation, identifying existing activities that could be delivered remotely with minimal changes. As the pandemic went on, SCD also created new resources specifically for a remote audience and adapted existing activities where appropriate, using our evaluation framework to ensure these activities continued to meet the aims of the in-person programme. This paper presents the process through which this was achieved, some of the benefits and challenges of remote engagement and the plans for 2021 and beyond.
\end{abstract}

\section{Introduction}

The Science and Technology Facilities Council (STFC), part of UK Research and Innovation (UKRI), is responsible for supporting, co-ordinating and promoting research, innovation and skills development in areas ranging from the largest astronomical scales to the tiniest constituents of matter [1]. STFC's Public Engagement vision is of a society that values and participates in scientific endeavour [2] and to achieve help this, Public Engagement is one of STFC's six strategic themes [3]. STFC also has a dedicated public engagement team with managers at each of the three largest sites and its headquarters. "Big Data and Computing" is one of their five key outreach themes [4].

STFC's Scientific Computing Department (SCD) is distributed across two STFC sites and manages high performance computing facilities, services and infrastructure, supporting some of the UK's most advanced scientific facilities.

SCD works across a number of large facilities at both the Daresbury Laboratory in Cheshire and the Rutherford Appleton Laboratory in Oxfordshire (such as the UK Worldwide LHC Computing Grid (WLCG) Tier 1 [5] and the JASMIN Super Data Cluster [6]), across a large number of scientific software packages (such as the materials modelling code CASTEP [7] and The HSL Mathematical Software Library [8]) and has close links with other computing centres around the UK, particularly including the Hartree Centre [9, 10].

Due to the global COVID-19 pandemic, SCD has pivoted its public engagement programme to be entirely remote for the foreseeable future. This paper presents the process through which this was achieved, the plans for 2021 as well as some benefits and challenges of remote engagement.

\section{Public Engagement pre-COVID-19}

SCD has a long and rich history of delivering face to face public engagement and outreach, both on site and in public places, as part of the wider STFC programme. One of the unique qualities of STFC's public engagement programme is providing school children and members of the public a chance to experience "big science" for themselves, by participating in a wider visit to one of STFC's high-tech laboratories and facilities [11]. These visits often involve a large number of visitors being hosted by STFC for a day of facility tours, hands-on workshops and talks delivered by our staff, see Fig. 1 and Fig. 2. 


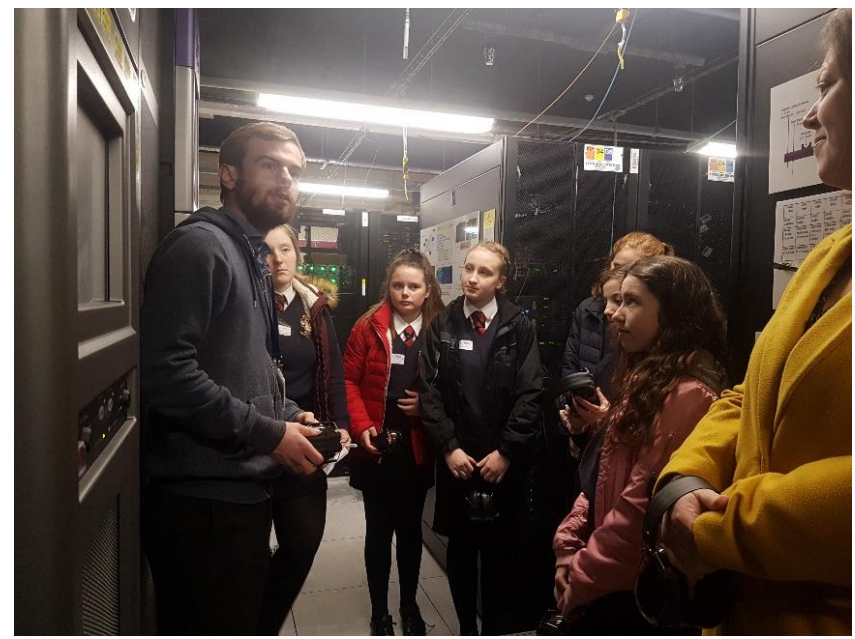

Fig. 1. A Data Centre tour in progress in 2019.

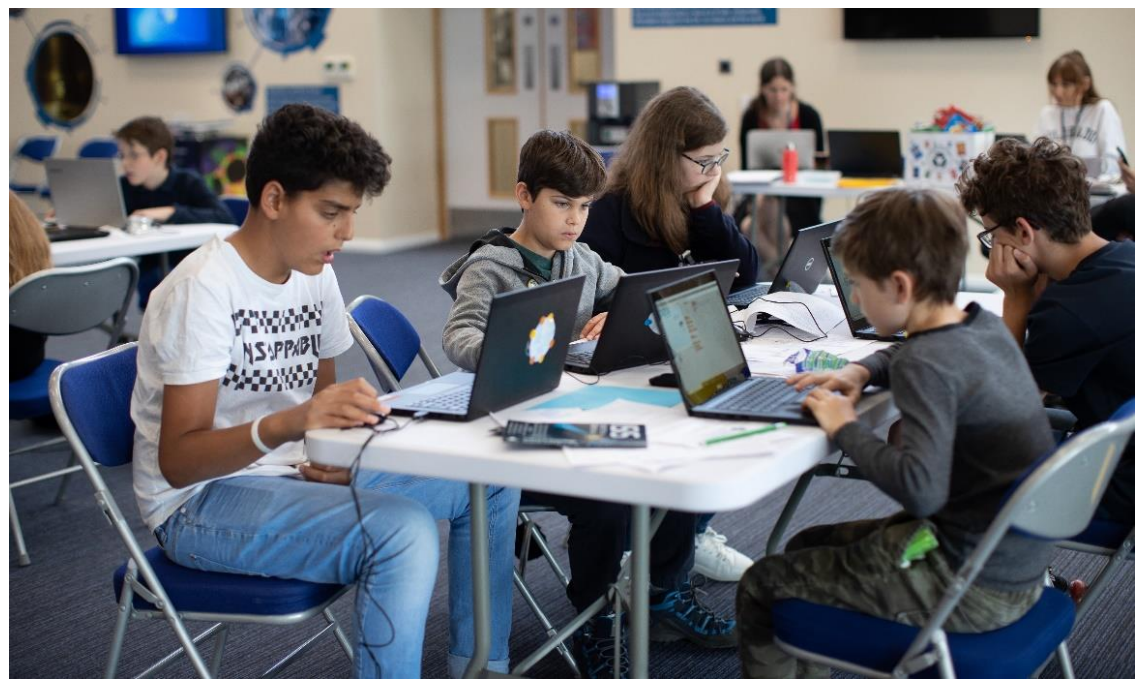

Fig. 2. A programming challenge in progress in 2019.

While this presents a unique and exciting experience for participants, it can limit the reach of STFC's Public Engagement efforts to those who have the means and ability to travel to one of the sites. STFC recognises this and has long had a strategic aim to improve the reach of its public engagement [2] by seeking to do more with audiences geographically remote from an STFC campus or other substantial STEM organisation, as well as increasing the proportion of our activities that reach low science capital [12] audiences.

In practice, it proved difficult to devote the necessary time and effort needed to get a significant remote programme off the ground whilst at the same time continuing with the ongoing programme of Public Engagement and its continuous improvement. There was also a reluctance and lack of technical familiarity for remote engagement from STFC's key audience before the pandemic. Despite this, STFC as a whole had recent successes in extending its geographical reach and SCD had started to think about engaging with remote schools before the pandemic, through a collaboration with another STFC site, the Boulby Underground Laboratory. Due to Boulby's location in a working mine, in-person visitors to the laboratory were of necessity rare (and children are not permitted underground for safety reasons), and so Boulby had developed techniques and expertise in remote engagement. 


\subsection{Enabling access; Travel bursaries and the Wonder Initiative}

STFC's Wonder initiative [13] is a long term commitment by STFC to audience-driven public engagement with communities in the most socioeconomically-deprived areas of the UK. Working with communities to understand and overcome barriers to engagement is a core part of STFC's PE mission, and many projects (both before and during the pandemic) work with community groups and other organisations to achieve this end. There has been a shift in some of these barriers during the national lockdowns - for example, before the pandemic, a key barrier was the cost of travelling to one of our laboratory sites. To begin to lower this barrier, STFC has used bursaries to cover the transport costs of schools located in areas of higher socioeconomic deprivation, or with a high proportion of students with free school meals. Understanding the new barriers that exist in a world of remote engagement is an important - and ongoing - part of our public engagement programme.

\subsection{Working with others, Boulby Underground Laboratory and the Remote ${ }^{3}$ Project}

The Remote ${ }^{3}$ or "Remote sensing by Remote schools in Remote environments" project is a collaborative effort between the University of Edinburgh, STFC's Boulby Underground Laboratory and STFC's Scientific Computing Department which was initiated in 2019 and funded by an STFC Spark Award, with the aim of delivering muchneeded STEM engagement to some of the most remote areas of Scotland. As the project title suggests, it was created as a remote project. Students from these areas are underserved in provision of STEM engagement compared to their urban counterparts, which is believed to be a contributing factor in low rate of progression to Higher and undergraduate education.

The project is aimed at S1-S3 students in 10 Scottish high schools (students ages 11-14). Teams of 4-6 students are assembled per school and over a 14-week period they design, build \& program a miniature Mars Rover, sharing their progress with their peers, teachers and families. This is then sent to the Boulby Underground Laboratory to explore the STFC Mars Yard, $1.1 \mathrm{~km}$ deep underground. This project has been constructed with an awareness of the specific skills and attributes in mind, seeking to inspire innovation and creative design, develop digital skills, encourage team-work, team-building and oral and written presentation skills in a diverse environment, and provide awareness of the remarkable on-going front-line scientific activity taking place in the UK and overseas.

To demonstrate the feasibility of the project, a trial project ran during RAL summer coding week in 2019, see Fig. 2 and Fig. 3. Twenty pupils aged from 8-14 were split into teams of 4 and at the end of the week they saw their Mars rovers remotely via zoom at the STFC Mars Yard at Boulby taking data and exploring the terrain. They then presented their findings to their parents and peers as well as STFC senior management.

The project began in early 2020, with a launch event with schools in early March - student teams were due to begin their projects as the first national lockdown began.

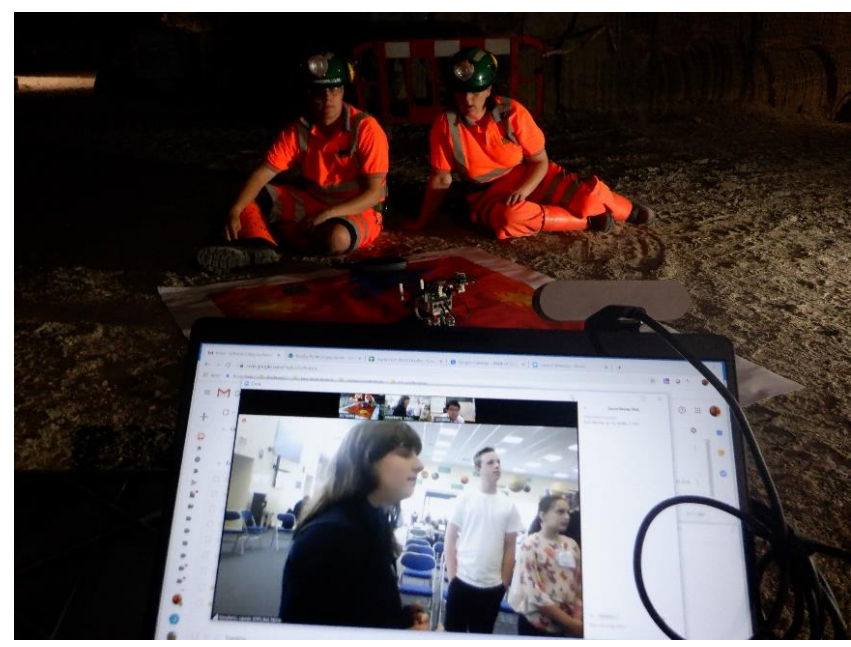

Fig. 3. The 2019 pilot event for Remote ${ }^{3}$, participants are watching their remote rover in the Mars Yard. 


\section{COVID-19 in the UK}

By the middle of March 2020, the vast majority of SCD staff started to work remotely from home, following the UK government's guidance to work from home if possible [14]. Access to STFC sites was limited to those working on and supporting COVID research. Less than a week later schools were closed with immediate effect [15], switching to remote learning where possible. This was to be the case for the foreseeable future, and as a result STFC's planned summer programme of in person Public Engagement, including a large scale public open week at Daresbury, was cancelled.

Since then, the UK public has faced a series of local and national restrictions on unnecessary social contact. At times of national lockdown, the strictest measures enforced to date in the UK, the public was told they must not leave the house except for a limited number of "reasonable" justifications [16].

\section{Remote Public Engagement}

With the summer programme cancelled, and not knowing when in person Public Engagement events would be feasible again, the SCD PE committee sought to redirect its efforts to devising remote activities and improvising a new Public Engagement programme. Remote PE brings its own challenges, particularly in areas such as safeguarding and evaluation, as well as a simple lack of equipment at home and reaching those who do not have good data connections, or access to a laptop / desktop device - an estimated $9 \%$ of families do not have access to a laptop, desktop or tablet at home [17].

\subsection{Initial Efforts}

The committee's immediate attention turned to highlighting existing standalone resources, such as the Hour of Code ${ }^{\mathrm{TM}}$ [18], a nationwide initiative to introduce millions of students to one hour of computer science and computer programming.

The first event SCD held during lockdown was a virtual version of the monthly RAL coding club. Normally a four hour session, the virtual version lasted two hours via video conferencing, starting with introductions and challenge setting. The children then worked on their individual projects, with mentors staying online to help as necessary. At 16:00 everyone was asked to sign back on to do a virtual "show-and-tell". Due to SCD's recurrent interactions and strong established relationships with the cohort, this event was able to make use of video feeds and screen share from its participants. The event helped the committee to gauge the speed at which participants can achieve goals during virtual sessions, their differing attentions spans, and helpful features of Zoom, including chat and reactions.

The committee also sought to interact with the wider efforts of the STFC PE and Social media teams, to provide content for a computing themed entry in the STFC's Science at Home Campaign [19]. This led to SCD's first remote activity of the pandemic, a challenge to recreate the Flexipede [20], which was one of the first ever computer-generated animated characters, created by Tony Pritchett in 1967, who had strong links to the ATLAS Computer Laboratory (now RAL). The option to "remix" an existing recreation [21], created especially for this challenge in Scratch, was also given.

\subsection{Subsequent Events}

Whilst these initial efforts were ongoing, the SCD PE committee worked to develop an effective autumn programme of public engagement events that could be delivered remotely. This was aided by the recent formalisation of the aims of each activity using the "Generic Learning Outcomes" (GLOs) evaluation framework [22], which ensured the new or adapted activities continued to meet the aims of the in-person activity.

\subsubsection{Remote Data Centre Tours}

The main GLOs of a face-to-face data centre tour are: understand some of the common terms used in High Performance Computing; be able to give examples of the speed / performance / memory of the Machines; be able to describe what a data centre looks like. A remote data centre tour, see Fig. 4, also seeks to meet these objectives through the use of a detailed SketchUp [23] model of an STFC data centre. To help ensure this, a remote tour is delivered by the same tour guides and in much the same style as a physical tour. 
A benefit of being remote is that the capacity of the tour is no longer limited by the ratio of non-staff to staff and the number of members of the public who can be in the data centre at the same time. As such, a remote tour is able to reach significantly more people with a reduction in the staff-time taken to deliver the tour. For example, whilst a physical tour would be limited to 6 per trained staff member, up to a maximum of 24, remote tours have been delivered by a single staff member to over 350 people in the same time as a physical tour would have taken place.
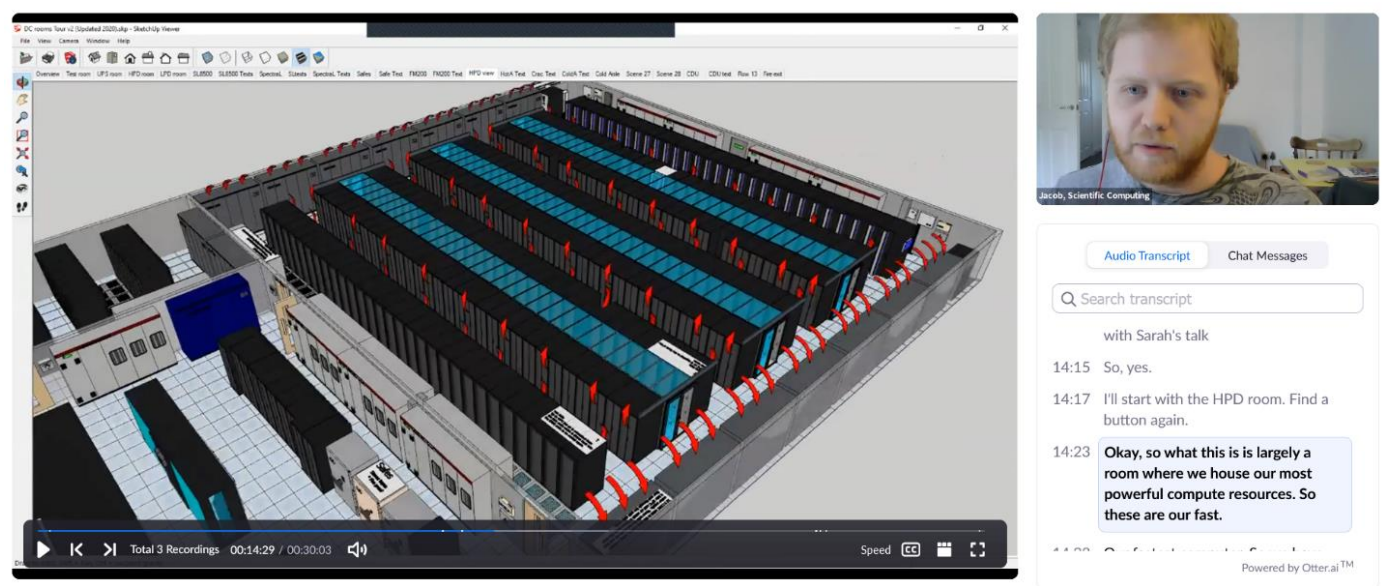

Fig. 4. A virtual data centre tour in progress.

A significant challenge of a remote data centre tour is offering a smooth viewing experience to the viewer in a Zoom webinar format. Whilst the model itself will run relatively smoothly on a typical staff laptop, the tour cannot be shared over Zoom on a typical staff laptop without a significant drop in framerate. The drop in framerate would negatively impact the "be able to describe what a data centre looks like" GLO, as it would hinder the participants' ability to get a sense of how the different parts of the data centre shown relate to one other in physical space. Currently, only one of the approximately 20 data centre tour guides have access to a laptop capable of sharing the tour over Zoom at a reasonable framerate, and that laptop is a personal gaming laptop. Whilst recording a video of the tour might solve this challenge, feedback from these events has indicated that participants feel more welcome in the remote tour because it is not a video, but is being delivered live for them. Participants feeling welcome is itself a key GLO of all our activities.

\subsubsection{Python Workshop}

As part of the STFC work experience programme, SCD usually supports the Public Engagement team to run a 3-day, face-to-face, introduction to python workshop at RAL [24] - usually attended by around 15 students. Obviously this was not possible in 2020, and as such the SCD PE committee commissioned a three-month project to rework the existing material [25] into something that could be delivered remotely as a pilot event. This project was funded by SCD and delivered by a computing apprentice.

JupyterHub Notebooks hosted on the STFC Cloud [26] was chosen as the new format for the workshop, see Fig. 5 , so that participants would not have to make use of any software beyond a web browser (i.e. there was no need for them to install python). The remote version was much shorter than the face-to-face version, being delivered in four, one hour sessions. As such, and due to the technical limitations of being unable to use pygame in a JupyterHub Notebook in a web browser, the remote version focused on the introduction to python. Much like the face-to-face version the remote version was a taught exercise led by a member of staff and supported by 14 other members of staff, who were on hand to help to debug the schoolchildren's code via the chat function. For safeguarding, participant video, audio and screen-sharing was disabled. 


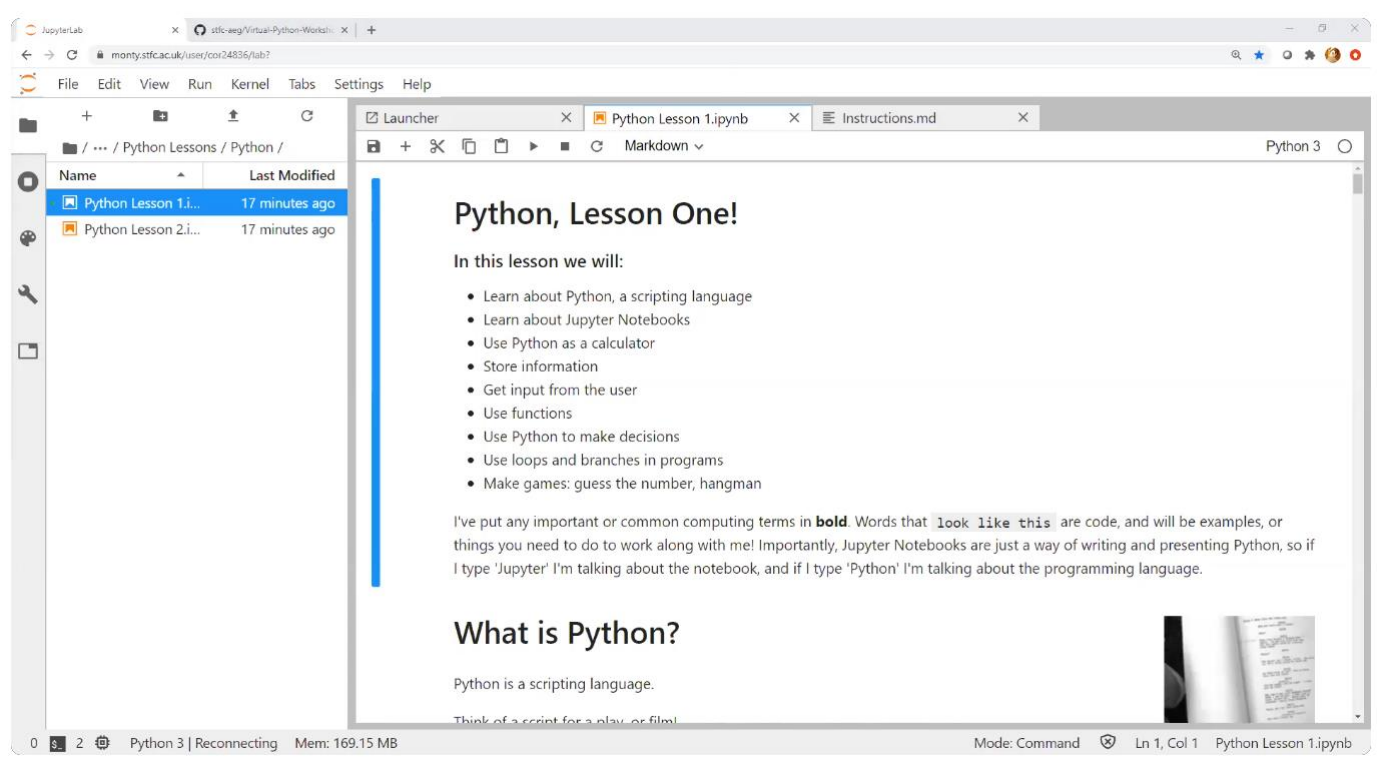

Fig. 5. The python workshop content as a Jupyter Notebook.

The remote nature of the event allowed it to reach 78 students, many more than is possible in the face-to-face version due to constraints on available computers and physical space. In the future, the material will be extended to allow it be a standalone resource that anyone can work though in their own time. The Jupyter Notebook platform will soon be delivered as a service by the STFC Cloud, allowing the SCD PE committee to build more engagement activities on top of it, and was successfully used to deliver a remote Particle Physics Masterclass [27] in March 2021, using LHC data.

\subsubsection{Work Experience}

In addition to the introduction to python workshop described above, work experience in 2020 took the form of a series of interactive webinars over the course of several months, focussing on different areas of STFC's work, and different career pathways and skills. These were very well received and again needed a smaller staff time input to reach a large number of students. The in-depth, immersive experience of work experience placements at the labs, however, is hard to replicate in a webinar format.

\subsubsection{Saturday Coding}

Saturday Coding has continued virtually on a monthly basis throughout the pandemic. The session times have remained at two hours duration, and there are definite challenges of holding these events remotely: it is harder to keep the participants on task, and harder to provide quick and effective support remotely. It has, however, encouraged the participants to develop the important skills of explaining the problems they are facing quickly and clearly, and supporting one another remotely.

\subsubsection{Ada Lovelace Day}

In October 2020, SCD hosted a remote version of its popular Ada Lovelace Challenge Day [24]. The event was held over Zoom and started with an introduction to physical and virtual [28] Arduino microcontrollers, as well as block based programming languages (such as Ardublock [29]). The face-to-face version had the GLOs that participants would understand coding and hardware, and the virtual Arduinos allowed the event to continue to meet this aim.

As schoolchildren were in the classroom for this event [30], each school was assigned a breakout room and multiple STFC staff members to help debug hardware and software issues. Each school had at least one Zoom video connection 
into the breakroom, with a teacher and two members of staff always present. Within a school, the schoolchildren were split into teams of 4 and each team started work on designing and building hardware and software based replacements for damaged systems, see Fig. 6, of the Ada Lovelace, the first ship to Mars carrying a crew.

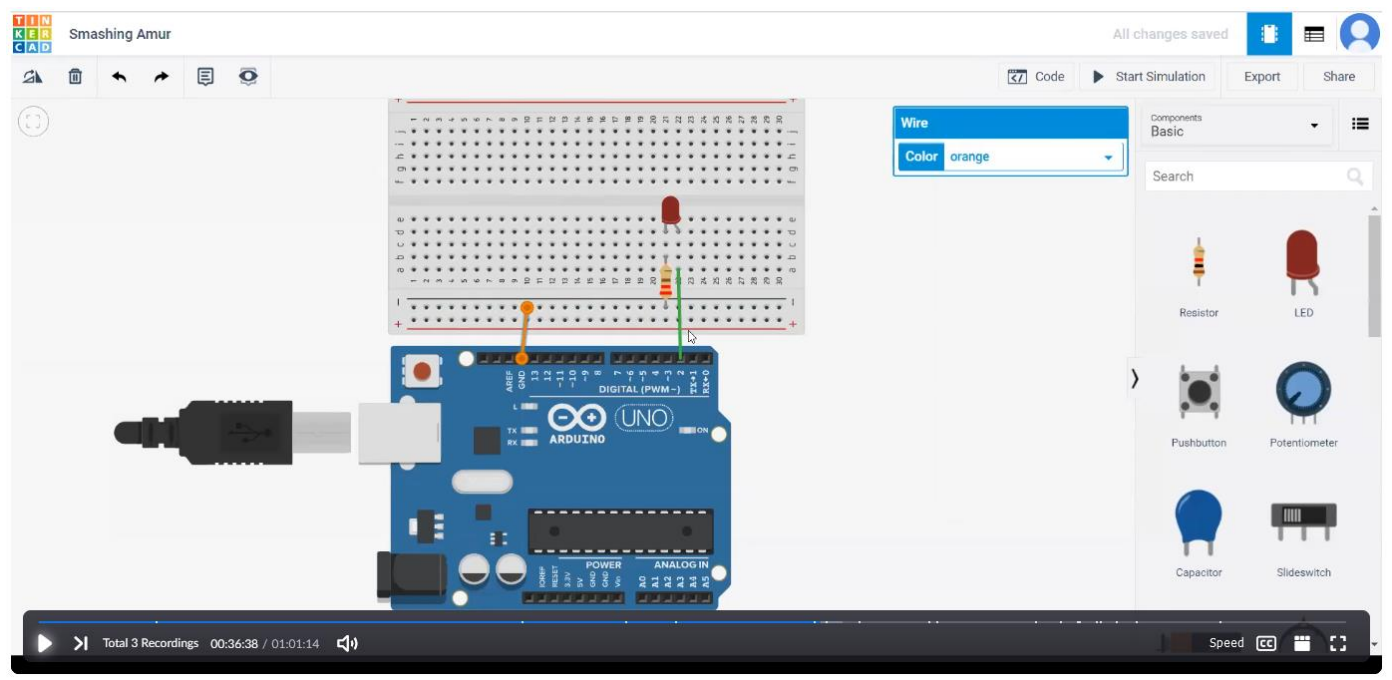

Fig. 6. A "Red Alert" system being built using a virtual Arduino.

To facilitate this event, some schools were sent physical Arduinos and a set of electronic components, as well as an electronic resources pack enabling them to replicate the day in the future on their own. The challenges were reworked to exclude any components that might overheat and cause even minor injury, as trained STFC staff would not be physically present with the components to ensure safety.

One of the benefits of its remote nature meant the event was able to reach over 350 schoolchildren, from a more geographically diverse area, instead of the approximately 40 schoolchildren, from the relatively near-by area, that the physical event can cater for. Feedback from both teachers and parents was excellent, with one parent writing to say: "Just wanted to report that my daughter has just got home from school where she has spent all day working remotely with RAL colleagues on some coding challenges. She absolutely loved it and said that she learned tons and all those involved were really nice."

There were challenges with the remote event, however. Some schools only had one video connection to the breakout rooms but had multiple classrooms worth of schoolchildren in the event and this caused delays while information was relayed by teachers. Going forward, SCD's remote events with schools will ensure one video connection per classroom. Some schools had technical problems with installing drivers and the Arduino IDE/Ardublock on school hardware, which was necessary to work with the physical Arduinos. Going forward, the leads of an SCD remote event with schools would arrange a pre-event meeting to debug these technical issues before the event itself. Future remote Ada Lovelace Challenge Days are also likely to make more use of the virtual Arduinos, as opposed to physical Arduinos.

\subsubsection{Remote Re $^{3}$}

Although Remote ${ }^{3}$ was conceived as a remote project from the start, the national lockdown and school closures necessitated a rapid re-consideration and adaptation of the planned programme. The school teams did not have access to physical Mindstorms, and often did not have reliable internet access at home. The project drew heavily on Boulby's experience and expertise of remote engagement to adapt the programme appropriately

The project stayed in touch with the teachers, offering help where needed and working with them, Boulby and the Scientific Computing Department to develop a series of online coding events which captured the core values of Remote $^{3}$, delivered weekly over 10 weeks. Each week introduced a new coding challenge, with participants creating solutions which were then demonstrated at subsequent events. Each event also featured a short talk or tour highlighting a particular aspect of science in remote environments, linking this back to the challenges the participants were 
undertaken to provide an aspirational context. The initial audience was expanded to more than just the Scottish schools, but also others around RAL and Boulby, with over 150 participants.

Sharing good practice and lessons learned during the pandemic, the Remote ${ }^{3}$ project was highlighted at a SUPA (Scottish Universities Physics Alliance [31]) showcase event. The project website was launched during national coding week and took part in the Big Bounce Festival, organised by the Institute of Physics, during which a coding workshop over a two day period was delivered.

In addition a Remote ${ }^{3}$ short story was developed for a much younger age group, which was delivered with great success to a wide range of audiences: from children to British Embassy staff in China, where it was a big hit. A series of curriculum-linked short activities which schools and uniform groups can undertake independently is also being worked on.

\subsubsection{Glow Your Own coding workshops}

In partnership with IF Oxford, RAL's local Science and Ideas Festival, and Fusion Arts (an Oxford-based charity that devises and delivers creative projects in the local and wider community), SCD supported and led a series of coding workshops using Arduinos, culminating in participants creating their own coded Christmas Lanterns. SCD provided support to enable IF Oxford to supply the community with physical Arduinos, removing the price barrier for those living in areas of higher socio-economic deprivation in Oxford. In addition to this, the link with the arts resulted in a high proportion of participants who had never coded before. The feedback was again extremely positive: "I'm astounded by the ambition you have with these series of workshops and how brilliantly well are explaining everything and making it all work for everyone. It's complex stuff, new to us and yet, here we all are, making it all work, with your help. Brilliant teamwork, great patience and dealing with the video and chat, all at the same time. BIG THANK YOU!!" The pacing of these workshops was very difficult to judge over Zoom, with a wide range of participants, but the project was so successful that it has been revived to take forward over the coming year.

\subsubsection{PE Induction for staff}

After running SCD's first PE induction in 2019 [32], a second workshop was delivered in October 2020 in a remote fashion. This time, it focused on the new remote activities developed earlier in the year, the JupyterHub based work experience workshop and the remote Arduino activity. The participants were introduced to common problems faced by students of both activities and how to debug and solve them. Following feedback from the previous induction, this time the workshop was open to everyone in SCD, not just early career staff. Evaluating the workshops effectiveness and planning next steps is very important: to this end, the participants were asked to rate the induction as well as suggest improvements and follow up training.

The delivery of this event benefited from the lessons learnt from the python workshop and Ada Lovelace Challenge Day, and many of the issues faced in those events were not repeated. Due to its virtual nature the number of breaks in the session was increased to avoid zoom fatigue, and as such the number of topics covered was reduced accordingly.

\subsection{Benefits}

Fortunately, in many ways computing lends itself to remote delivery more easily than other subjects might and switching to a purely remote programme of public engagement has brought benefits to SCD's public engagement efforts. One benefit is that the programme is no longer limited by the physical constraints of an STFC site, nor limited by geography to those who have the means and ability to travel to an STFC site. Without the cost of SCD staff going into schools to deliver engagement, the SCD PE Committee has been able to send resources, such as Arduinos, to schools so that they can participate in events remotely. These resource then remain at the school, allowing teachers to redeliver the activity themselves in future years.

Given that the majority of staff within SCD, and STFC as a whole, are now working remotely from home, the pandemic has also encouraged more interaction between the public engagement efforts of STFC two largest sites, the Rutherford Appleton and Daresbury Laboratories. The pandemic has also acted as a catalyst for the SCD PE Committee to adapt existing resources and develop new resources such that they can be delivered remotely. Whilst these resources do fill a short term need for remote public engagement, they also help achieve the longer term aims of reaching a more geographically diverse audience. When in-person public engagement returns, given the clear benefits of remote engagement, the programme is not foreseen to return to being purely in-person. These remote activities will continue to be a part of a blended in-person/remote hybrid programme of Public Engagement, though it is not yet clear what proportion of activities will be remote going forward. Over the coming months we will assess which types of 
activity benefit most from being delivered remotely, as opposed to in-person delivery, and will adjust the balance of the programme accordingly

Times of national lockdown and school closures are causes of stress for the public, teachers and parents. With people confined, the opportunity to visit places like STFC's big facilities virtually is welcome. With schools closed, the fact STFC can reach out with resources is perhaps more welcome

The ubiquity of video conferencing in the public's everyday life as a result of the pandemic has made remote public engagement easier. Before the pandemic, it was easier for teachers and schoolchildren to come onto site then it was to arrange a video call on a suitable, well-used and well-understood platform. The pandemic resulted in video conferencing software becoming much more of a necessity for organisations (as an example STFC did not have the technical capability to host large scale webinars before the pandemic - the necessary license for which was only acquired after mass working from home began). The software itself has also become much more feature-full in the areas of privacy and security, and people are far more confident now using this kind of software.

\subsection{Challenges}

Remote Public Engagement brings its own set of challenges. Some of these challenges are a result of the uncertainty of life in a global pandemic, however some are specifically challenges for remote engagement.

When schoolchildren started to return to the classroom in summer 2020, STFC as a whole saw less of a demand for the public engagement programme as teachers had to focus on catch up on progress through the curriculum, which had been lost during the first lockdown,. This disproportionately affected disadvantaged pupils [33], who tend to have lower science capital and as such are one of STFC's key PE audiences.

Planning remote public engagement events against the backdrop of uncertainty, not knowing whether the majority of schoolchildren will be in classrooms or home-learning, is also more challenging. As SCD and STFC plan their public engagement programmes for 2021, plans must be made not only for remote events, but also remote events that will work well regardless of whether the schoolchildren are physically together in a classroom, learning from home, or a mix of the two.

Evaluating remote public engagement is inherently a different problem to evaluating in-person engagement, regardless of a pandemic. STFC collects evaluation data from all its events. When these events were in-person, individual participants would often evaluate the event as the event was ending, while still on site. This cannot be enforced in a remote setting. Participants with a negative view of an event may leave early, before any evaluation takes place, and those who do provide feedback after an event has ended may be those who have extreme views of the event. In a remote setting evaluation of events is also reduced to per zoom connection, which is typically per household or classroom. With the loss of this fine grained information, often a consensus view of the event is reported and it is more difficult to get accurate demographic information, such as the number of girls engaged, which is a key STFC demographic [2]. As such, evaluation may skew to the positive and it is difficult to make direct comparisons of how the programme has changed due to the shift to remote engagement.

The sudden shift in the programme from in-person to remote engagement also makes comparisons of the evaluation data from this year to previous years difficult. SCD was set to commission a project to evaluate its in person programme, to ensure it was meeting its aims and to improve the programme as a whole.

Remote public engagement also has its own challenges to safeguarding. With face to face engagement, it is often trivial to ensure a single member of staff is never left alone with a schoolchild in a private space by simply avoiding areas with little to no footfall. However, with remote public engagement, every Zoom call is essentially a private place. As such STFC took technical and procedural steps to ensure safeguarding requirements were still met. These included: disabling participant video and screen sharing for events such as the python workshop; and ensuring any breakout room has at least two members of staff in them at all times.

Getting new people involved with the STFC public engagement programme has also suffered during lockdown, though SCD's computing activities do have higher proportion of new people involved due to its culture of PE [32] and the virtual PE induction. During the pandemic it can be difficult for some staff to work effectively from home, due to childcare, wellbeing or other concerns, let alone deliver remote public engagement. In the future, staff who have found it difficult to work from home will likely return to site - which may reduce some of the barriers that are currently stopping them getting involved in PE. Conversely, more staff may start working mostly from home, which may place new barriers on them getting involved with in person PE. The timing of any future PE training will have to take into account these new working patterns, perhaps co-locating the training with other events that require staff to attend a STFC site.

Also, remote events tend to require fewer members of staff to deliver and, with the added difficulty of delivering online, the temptation is to fall back to those with experience of delivering the session rather than on new members of 
staff. There are also technical limitations. A virtual tour is computationally expensive to run, limiting who can deliver it to those with high end gaming machines, which are typically personal devices.

\section{Future}

The SCD PE Committee is aware that many of the new activities created require a level of access to the internet and technology that not everyone in the UK will have, especially at home. This "digital divide" [34] still limits the reach of the engagement SCD conducts. As the programme for 2021 takes shape, SCD is keen to lower as many technological barriers as possible. This could be through reducing the number of screens/devices needed for optimum participant in events, sending the necessary hardware to participants, or removing the need for a computer/internet connection entirely. The STFC Public Engagement Team is currently working on the development of a data logger project, where hardware (Raspberry Pis and sensors) will be provided to families through (e.g.) libraries and community groups. STFC is also working with our local Computing at School to improve accessibility for remote events.

One way to remove the need for a computer/internet connection entirely would be to leverage the paper based "Unplugged Computing" activities recently created at CERN, such as the "Introduction to Programming" activity (where a grid, maze and arrow cards are used by children to navigate a robot between two squares) and the "What's Inside a Computer" activity (where participants learn about some core components of computers) [35]. Indeed, SCD had started to look into how these could be incorporated into the Daresbury Open Week before it was cancelled.

Whilst 2020 was an exercise in improvisation, 2021 has been planned from the outset to be a year of mostly remote public engagement. The SCD PE committee will build on the lessons learnt from the initial events discussed in Section 4 to deliver a programme of engagement and endeavour to ensure the schools programme will work well regardless of whether schoolchildren are in classrooms, at home, or a mix of both.

\section{Conclusions}

Since the start of the pandemic, remote Public Engagement has been the norm for SCD and STFC as a whole. SCD was forced to abandon an extensive planned programme, including the Daresbury Open Week (into which significant effort had already been put), alongside altering the day-to-day working methods of the majority of its staff. The year was initially an exercise in improvisation with existing activities quickly identified that could be delivered remotely with minimal changes. During this time, SCD also created new resources specifically for a remote audience and adapted existing activities where appropriate.

Throughout the process, SCD has relied on the STFC Evaluation Framework and the concept of "Generic Learning Outcomes" to ensure the new remote events continue to meet the same objectives as the face-to-face portfolio. As a result SCD now has a portfolio of remote activities and, whilst remote public engagement brings both benefits and challenges, SCD firmly believes the remote activities developed and those that will be developed during the pandemic will remain a key part of the public engagement programme when staff and visitors are able to return to the sites. This all contributes to STFC's long held strategic aim to extend the reach of its public engagement to audiences geographically remote from an STFC site.

Moving forward, SCD will learn from the pilot events this year to deliver an improved programme in 2021, which is expected to be another year of remote engagement.

\section{References}

1. STFC, "STFC Vision - Science and Technology Facilities Council," 28 February 2019. [Online]. Available: https://stfc.ukri.org/about-us/our-purpose-and-priorities/stfc-vision/. [Accessed 9 February 2021].

2. Science and Technology Facilities Council, "Inspiring and Involving," [Online]. Available: https://stfc.ukri.org/files/corporate-publications/stfc-public-engagement-strategy/. [Accessed 9 February 2021].

3. STFC, "Strategic Context \& Future Opportunities," 2018. [Online]. Available: https://stfc.ukri.org/files/corporate-publications/strategic-context/. [Accessed 9 February 2021].

4. STFC, "Inspiring Stories - Science and Technology Facilities Council," 13 June 2018. [Online]. Available: https://stfc.ukri.org/public-engagement/inspiring-stories/. [Accessed 9 February 2021].

5. GridPP, "RAL Tier1 - GridPP Wiki," 15 December 2019. [Online]. Available: https://www.gridpp.ac.uk/wiki/RAL_Tier1. [Accessed 9 February 2021]. 
6. CEDA, "CEDA | JASMIN," [Online]. Available: https://www.ceda.ac.uk/services/jasmin/. [Accessed 09 February 2021].

7. CASTEP, " Main/Home Page," 10 February 2020. [Online]. Available: http://www.castep.org/. [Accessed 9 February 2021].

8. HSL, "HSL Mathematical Software Library," [Online]. Available: http://www.hsl.rl.ac.uk/. [Accessed 9 February 2021].

9. SCD, "SCD About Us," [Online]. Available: https://www.scd.stfc.ac.uk/Pages/About-Us.aspx. [Accessed 9 February 2021].

10. Hartree Centre, "Hartree Centre - Hartree Centre | Home," [Online]. Available: https://www.hartree.stfc.ac.uk/Pages/home.aspx. [Accessed 9 February 2021].

11. Science and Technology Facilities Council, "Where We Work - Science and Technology Facilities Council," 16 May 2017. [Online]. Available: https://stfc.ukri.org/about-us/where-we-work/. [Accessed 9 February 2021].

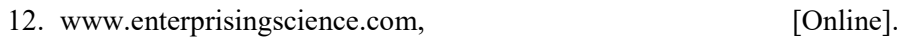

Available: https://www.stem.org.uk/sites/default/files/pages/downloads/Science-Capital-Made-Clear.pdf. [Accessed 11 February 2021].

13. "The Wonder initiative," [Online]. Available: https://stfc.ukri.org/public-engagement/wonder/. [Accessed 25 February 2021].

14. Prime Minister's Office, 10 Downing Street, "Prime Minister's statement on coronavirus (COVID-19): 16 March 2020 - GOV.UK," 16 March 2020. [Online]. Available: https://www.gov.uk/government/speeches/pm-statementon-coronavirus-16-march-2020. [Accessed 9 February 2021].

15. Prime Minister's Office, 10 Downing Street, "Prime Minister's statement on coronavirus (COVID-19): 22 March 2020 - GOV.UK," 22 March 2020. [Online]. Available: https://www.gov.uk/government/speeches/pm-statementon-coronavirus-22-march-2020. [Accessed 9 February 2021].

16. UK Government, "National lockdown: Stay at Home - GOV.UK," 1 February 2021. [Online]. Available: https://www.gov.uk/guidance/national-lockdown-stay-at-home. [Accessed 10 February 2021].

17. C. Commissioner. [Online]. Available: https:/www.childrenscommissioner.gov.uk/2020/08/18/childrenwithout-internet-access-during-lockdown/.

18. https://www.facebook.com/Code.org, "Hour of Code: Join the Movement," 2021. [Online]. Available: https://hourofcode.com/uk. [Accessed 9 February 2021].

19. Science and Technology Facilities Council, "Science and Technology Facilities Council on Twitter," 20 April 2020. [Online]. Available: https://twitter.com/stfc_matters/status/1252154041855025152. [Accessed 9 February 2021].

20. Computer History Museum, "The flexipede : a film drawn \& animated by computer | 102622004 | Computer History Museum," [Online]. Available: https://www.computerhistory.org/collections/catalog/102622004. [Accessed 9 February 2021].

21. Science and Technology Facilities Council, "Flexipede Animation on Scratch," 21 April 2020. [Online]. Available: https://scratch.mit.edu/projects/384035327/. [Accessed 9 February 2021].

22. Science and Technology Facilities Council, "Public Engagement Evaluation Framework," April 2017. [Online]. Available: [Accessed 9 February 2021].

23. Trimble, "3D Design Software | 3D Modeling on the Web | SketchUp," 2020. [Online]. Available: https://www.sketchup.com/. [Accessed 9 February 2021].

24. G. Corbett, G. Ryall, S. Palmer, I. Collier, J. Adams and R. Appleyard, "Public Outreach at RAL: Engaging the Next Generation of Scientists and Engineers," in Journal of Physics: Conference Series, 2015.

25. Science and Technology Facilities Council, "Release STFC Work Experience Computing Workshop: 2019 Release · stfc/WorkExp-CompWorkshop," 11 June 2019. [Online]. Available: https:/github.com/stfc/WorkExpCompWorkshop/releases/tag/v2019.1. [Accessed 10 February 2021].

26. Science and Technology Facilities Council, "Welcome to the STFC Cloud," 2016. [Online]. Available: https://openstack.stfc.ac.uk/. [Accessed 10 February 2021]. 
27. Science and Technology Facilities Council, "Particle Physics Masterclasses - Science and Technology Facilities Council," 31 October 2019. [Online]. Available: https://stfc.ukri.org/news-events-andpublications/events/general-interest-events/particle-physics-masterclasses/. [Accessed 10 February 2021].

28. Autodesk, Inc, "Tinkercad | Create 3D digital designs with online CAD | Tinkercad," 2021. [Online]. Available: https://www.tinkercad.com/. [Accessed 10 February 2021].

29. Science and Technology Facilities Council, "stfc/ardublock: ArduBlock is a Block Programming Language for Arduino.," 3 July 2020. [Online]. Available: https://github.com/stfc/ardublock. [Accessed 10 February 2021].

30. BBC News, "School figures show $88 \%$ of pupils were back for start of term - BBC News," 15 September 2020. [Online]. Available: https://www.bbc.co.uk/news/education-54164487. [Accessed 10 February 2021].

31. "SUPA: Scottish Universities Physics Alliance," [Online]. Available: https://www.supa.ac.uk/. [Accessed 25 February 2021].

32. G. Corbett, I. Collier and S. Palmer, "Public Engagement - More than just fun," in EPJ Web of Conferences, 2020.

33. J. Burns, "Coronavirus: Lockdown pupils are three months behind, say teachers," 1 September 2020. [Online]. Available: https://www.bbc.co.uk/news/education-53947982. [Accessed 11 February 2021].

34. C. Baker, G. Hutton, L. Christie and S. Wright, "COVID-19 and the digital divide - POST," 17 December 2020. [Online]. Available: https://post.parliament.uk/covid-19-and-the-digital-divide/. [Accessed 10 February 2021].

35. M. A. Pradillo, E. Badinova, A.-C. Chelba, H. M. Serans, N. Kane, S. Kriva and H. Short, "Unplugged Computing for Children," in EPJ Web of Conferences, 2020. 